\title{
Dielectric and thermal studies on gel grown strontium tartrate pentahydrate crystals
}

\author{
A FIRDOUS ${ }^{\dagger}$, I QUASIM, M M AHMAD ${ }^{\dagger}$ and P N KOTRU* \\ Department of Physics and Electronics, University of Jammu, Jammu 180 006, India \\ ${ }^{\dagger}$ Department of Physics, National Institute of Technology, Hazratbal, Srinagar 190 006, India
}

MS received 20 February 2009; revised 31 March 2009

\begin{abstract}
Results of dielectric and thermal studies on strontium tartrate pentahydrate crystals are described. The value of dielectric constant is shown to be independent of temperature till $360 \mathrm{~K}$ at all the frequencies (110-700 kHz) of the applied a.c. field. It increases abruptly achieving a peak value of 25.5 at $100 \mathrm{kHz}$; the peak value being strongly dependent on frequency. In the temperature range, $87<T<117^{\circ} \mathrm{C}$, the value of $\varepsilon^{\prime}$ falls suggesting a transition at around $100^{\circ} \mathrm{C}$ or so. The dielectric constant, $\varepsilon^{\prime}$, of the material is shown to be frequency dependent but temperature independent in the pre- or post- $T_{\mathrm{c}}$ range $87<T<117^{\circ} \mathrm{C}$, suggesting that the contribution towards polarization may be due to ionic or space charge polarization which gets eliminated at higher frequencies. The ferroelectric transition is supported by the results of thermoanalytical studies. It is explained that crystallographic change due to polymorphic phase transition may be occurring in the material, besides the change due to loss of water molecules, which leads to the dielectric anomaly at around $100^{\circ} \mathrm{C}$. Coats-Redfern approximation method is applied for obtaining non-isothermal kinetic parameters leading to calculation of activation energies corresponding to three decomposition stages of material in the temperature ranging from $379-1113 \mathrm{~K}$.
\end{abstract}

Keywords. Permittivity; polarization effects; strontium tartrate; thermal properties; dielectric properties.

\section{Introduction}

Growth of crystals from gels (Henisch 1973, 1988) is an inexpensive and unique method for growing crystals that show poor solubility in water. The salts of tartaric acid are found to exhibit a range of interesting physical properties such as ferroelectricity, piezoelectricity and optical second harmonic generation (Toress et al 1998; Fousek et al 1970; Ivanov 1984; Jona and Shirane 1993). Consequently they are used in transducers and in several linear and non-linear mechanical devices (Toress et al 1995, 1998, 2006; Fousek et al 1970; Ivanov 1984; Jona and Shirane 1993). This has led many investigators to grow single crystals of tartrate compounds and study their characteristics (Suryanarayan and Dharmaprakash 2000, 2002; Arora et al 2004, 2005). In the present study, we report the growth and characteristics of strontium tartrate pentahydrate (STP) single crystals, bearing the chemical formula, $\mathrm{SrC}_{4} \mathrm{H}_{4} \mathrm{O}_{6} \cdot 5 \mathrm{H}_{2} \mathrm{O}$, which shows that the crystal grown contains five coordinated water molecules. Arora et al $(2004,2005)$ reported the dielectric behaviour of strontium tartrate tetrahydrate bearing chemical formula, $\mathrm{SrC}_{4} \mathrm{H}_{4} \mathrm{O}_{6} \cdot 4 \mathrm{H}_{2} \mathrm{O}$. Strontium tartrate pentahydrate grown by us is different from the one grown by Arora et al not

\footnotetext{
*Author for correspondence (pnkotru@rediffmail.com)
}

only in respect of number of water molecules but also in terms of cell parameters, morphological development, thermal stability and FTIR spectroscopic results. As a result, the properties of the grown crystals such as mechanical properties, dielectric properties, magnetic properties (Arora et al 2004, 2005) and thermal properties (Rahimkutty et al 2001) are expected to be quite different from the crystals grown by Arora et al (2004, 2005) and Rahimkutty et al (2001). The details of experiments performed to grow STP from silica gel are described and discussed elsewhere. Very interesting results on indentation induced hardness testing of these crystals have been obtained by us and the same will be published elsewhere. In the present paper, results of studies on dielectric and thermal characteristics of this material are described.

\section{Experimental}

The single crystals of STP were prepared by single tube ionic diffusion through acidified silica hydrogel. The transparent glooming single crystals with well faceted habit faces were obtained within the gel columns. The single crystals of STP with habit faces (001), (011), $(01 \overline{1}),(100)$, and (210) were obtained. The dimensions of the single crystals ranged between $\left(7 \times 5 \times 2.5 \mathrm{~mm}^{3}\right)$ and 
$\left(21 \times 9 \times 7.5 \mathrm{~mm}^{3}\right)$. The crystals were found to have orthorhombic structure with the space group P212121. The unit cell dimensions as determined by using Powder$\mathrm{X}$ software are $a=9.47 \AA, b=10.96 \AA$ and $c=9.45 \AA$ with interfacial angles, $\alpha=\beta=\gamma=90^{\circ}$, which are different from the one reported by Arora et al $(2004,2005)$. For conducting dielectric studies of $\mathrm{SrC}_{4} \mathrm{H}_{4} \mathrm{O}_{6} \cdot 5 \mathrm{H}_{2} \mathrm{O}$, a single crystal with prominent (100) face was mounted between a pair of electrodes. The liquefied nitrogen was used to decrease the temperature of the sample holder nearly to $77 \mathrm{~K}$. However, the capacitance was intended to be measured from $80 \mathrm{~K}$, but the dielectric response between $80 \mathrm{~K}$ and $150 \mathrm{~K}$ was found to be practically nil. Therefore, the capacitance of the grown sample between (100) faces was measured in the temperature range, 150$450 \mathrm{~K}$. The sample was specially mounted on a threeterminal device holder. The measurements were recorded with the help of precision LCR meter model Agilent4284A and further automated by using a computer for the data recording, storage and analysis. The instrument directly provides the value of capacitance and dielectric loss $(\tan \delta)$. The dielectric constant $\left(\varepsilon^{\prime}\right)$ was computed using the relation

$$
\varepsilon^{\prime}=4 C t / 0 \cdot 0885 A,
$$

where $C$ represents the capacitance of the sample, $t$ the thickness, $A$ the area of the sample and $f$ the frequency of the applied a.c. field in Hertz. The single crystals of STP were characterized by using X-ray powder diffraction, $\mathrm{CH}$ analysis, scanning electron microscopy, FTIR spectroscopy and thermoanalytical techniques viz. TG and DTA. The powder XRD pattern of STP was obtained using Bruker D8 advanced X-ray diffractometer with $\mathrm{CuK} \alpha$ radiation $(\lambda=1.5406 \AA)$. The $\mathrm{C}$ and $\mathrm{H}$ contents in the crystals were determined by using Vario-EL-III CHNSanalyser. The FTIR spectra of the material in the range of $400-4000 \mathrm{~cm}^{-1}$ were recorded on the Bruker Vector 22 spectrometer using $\mathrm{KBr}$ pellet technique. The thermal behaviour of the grown crystals was recorded on the Shimazdu DTG 60 thermal analyser in nitrogen atmosphere at a heating rate of $10^{\circ} \mathrm{C} / \mathrm{min}$. The results of these characterization probes are reported elsewhere.

\section{Results}

\subsection{Dielectric studies}

Figure 1 shows the dependence of dielectric constant on temperature in the range $150-575 \mathrm{~K}$ under frequency of the applied a.c. field ranging between 100 and $700 \mathrm{kHz}$. The following points are noteworthy: (i) the dielectric constant is independent of temperature till near about $360 \mathrm{~K}$ at all frequencies, (ii) the dielectric constant abruptly increases and achieves a peak value at around $370 \mathrm{~K}$, after which it decreased. Though the values of $\varepsilon^{\prime}$ corresponding to a particular temperature decreases as the frequency of the applied a.c. field increases, the temperature at which the transition takes place is practically the same at all the frequencies considered here, (iii) the values of dielectric constant at $150 \mathrm{~K}$ for higher frequencies (600 and $700 \mathrm{kHz}$ ) of the applied a.c. field are 18.5 and 19.5 , respectively. Below this frequency, the value of dielectric constant is very nearly the same at about 20.4 which remains almost the same under the lower frequency range of 100-500 kHz. There is very little, almost negligible, change in the value of dielectric constant in the frequency range $100-500 \mathrm{kHz}$, (iv) except for the sudden variation in the value of dielectric constant at the transition points $(\sim 370 \mathrm{~K})$, it remains the same, before and after it in the entire temperature range of 150-360 and $380-575 \mathrm{~K}$ and $(\mathrm{v})$ the transition is sharper at higher

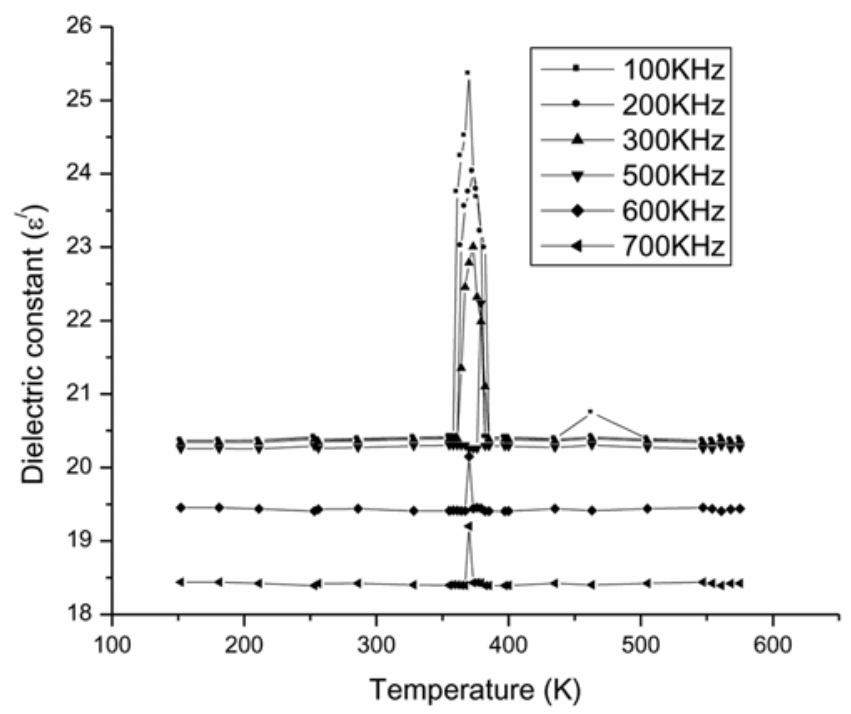

Figure 1. Graphical presentation of variation of dielectric constant with temperature.

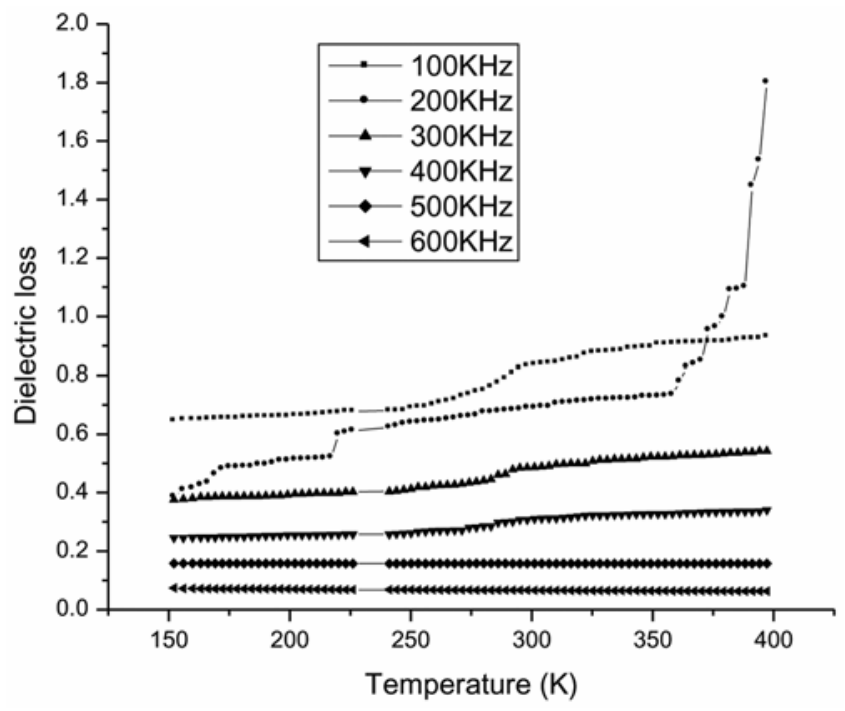

Figure 2. Graphical plot of variation of $\tan \delta$ with temperature. 
frequencies of 700 and $600 \mathrm{kHz}$ while it gets relatively diffused in the lower frequency range $(500 \mathrm{kHz}$ and below)

The dependence of dielectric loss on temperature under different frequencies as suggested by figure 2 may be briefly discussed as follows: (i) the dielectric loss at a particular temperature decreases as the frequency increases; its value at $150 \mathrm{~K}$ being $0.65,0.4,0.25,0.16$ and $0 \cdot 1$ at $100,300,400,500$ and $600 \mathrm{kHz}$, respectively. In other words, the dielectric loss at $150 \mathrm{~K}$ attains the highest value of 0.65 at the lowest frequency of $100 \mathrm{kHz}$ and about $0 \cdot 1$ at the highest frequency of $600 \mathrm{kHz}$, (ii) at higher frequencies of 500 and $600 \mathrm{kHz}$ the dielectric loss is independent of temperature in the range 150-400 K. However, dependence of dielectric loss on temperature does not remain independent of temperature at lower frequency of $400 \mathrm{kHz}$ and below once the temperature goes beyond $240 \mathrm{~K}$. Till $240 \mathrm{~K}$ it remains independent

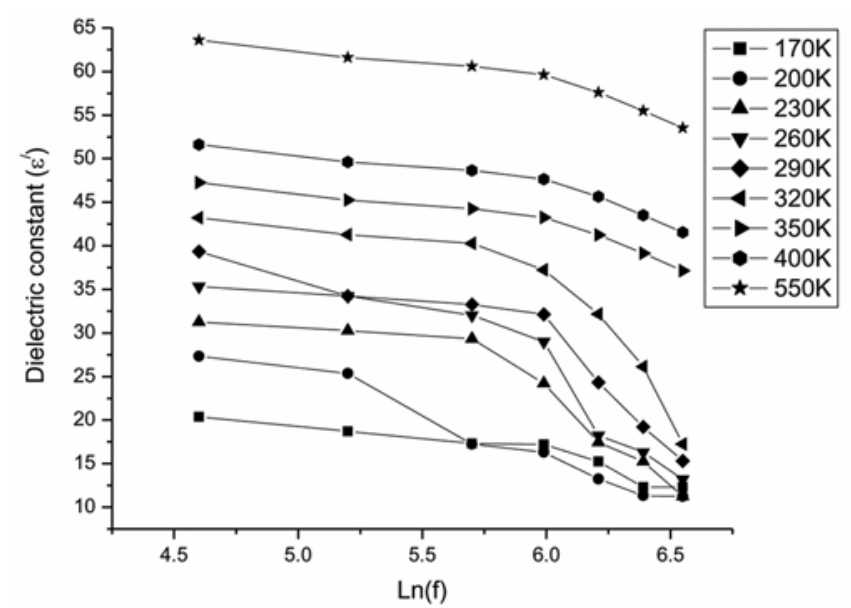

Figure 3. Plot of dielectric constant vs frequency.

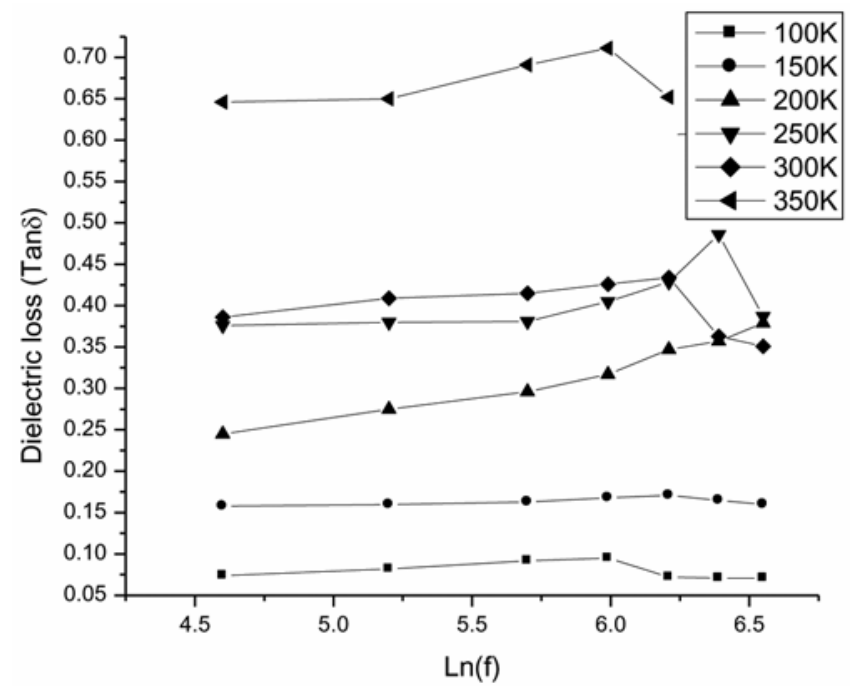

Figure 4. Plot of dielectric loss vs frequency. of temperature at all frequencies in the range 100$600 \mathrm{kHz}$.

The dependence of dielectric constant on frequency at different temperatures in the range $170-550 \mathrm{~K}$ is shown in figure 3 . The following points may be noteworthy: (i) the dielectric constant at a particular frequency is higher as the temperature is increased from $170 \mathrm{~K}$ to $550 \mathrm{~K}$, (ii) the general pattern of the curves suggest that the $\varepsilon^{\prime}$ decreases negligibly with increase in frequency at all temperatures in the range $170-550 \mathrm{~K}$ till the value of $\log f$ reaches $5 \cdot 75$. However, thereafter, $\varepsilon^{\prime}$ shows a decreasing trend at all temperatures in the range $170-550 \mathrm{~K}$.

Dependence of dielectric loss on frequency at different temperatures is revealed in a plot of dielectric loss vs log of frequency (figure 4) at different temperatures in the range $100-350 \mathrm{~K}$. It is clear that: (a) the dielectric loss is almost independent of frequency up to $\log f=6$ in the lower temperature range of $100-150 \mathrm{~K}$. However, the

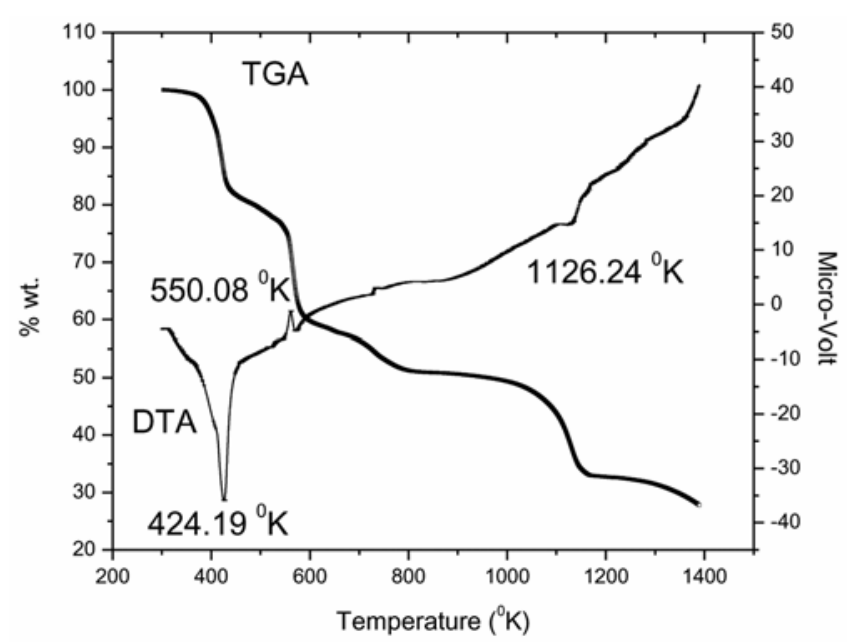

Figure 5. TGA and DTA of strontium tartrate pentahydrate

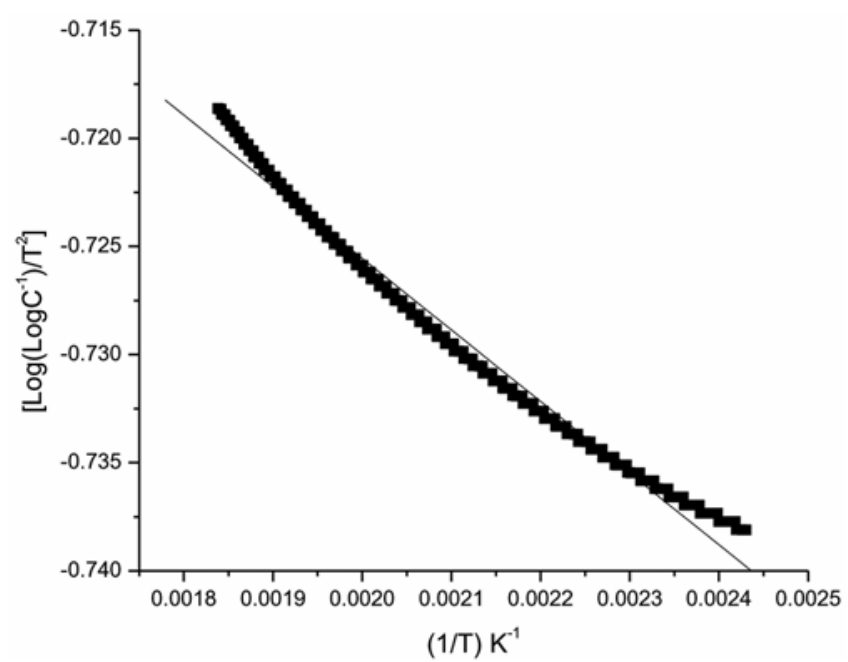

Figure 6. Plot of best fitting for stage 1 (379-445 K). 
value of $\tan \delta$ no longer remains independent of frequency at higher temperatures ranging from $200-350 \mathrm{~K}$ for frequencies $>\log f=5 \cdot 2$. Below this frequency $(\log f=4 \cdot 5-5 \cdot 2), \tan \delta$ is independent of temperature for all temperatures in the range considered here (100$350 \mathrm{~K})$ and (b) the dielectric loss, $\tan \delta$, attains highest values with the increase in temperature corresponding to all the frequencies considered here.

\subsection{Thermal studies}

The simultaneous TGA and DTA recorded for strontium tartrate pentahydrate in the temperature range 313$1473 \mathrm{~K}$ is shown in figure 5 . From figure 5 it is clear that the material is stable up to $378 \mathrm{~K}$. However, in three temperature ranges, $379-445 \mathrm{~K}, 547-797 \mathrm{~K}$ and $1039-1113 \mathrm{~K}$, the material loses three coordinated water molecules, two intrinsic water molecules and one intrinsic water molecule,

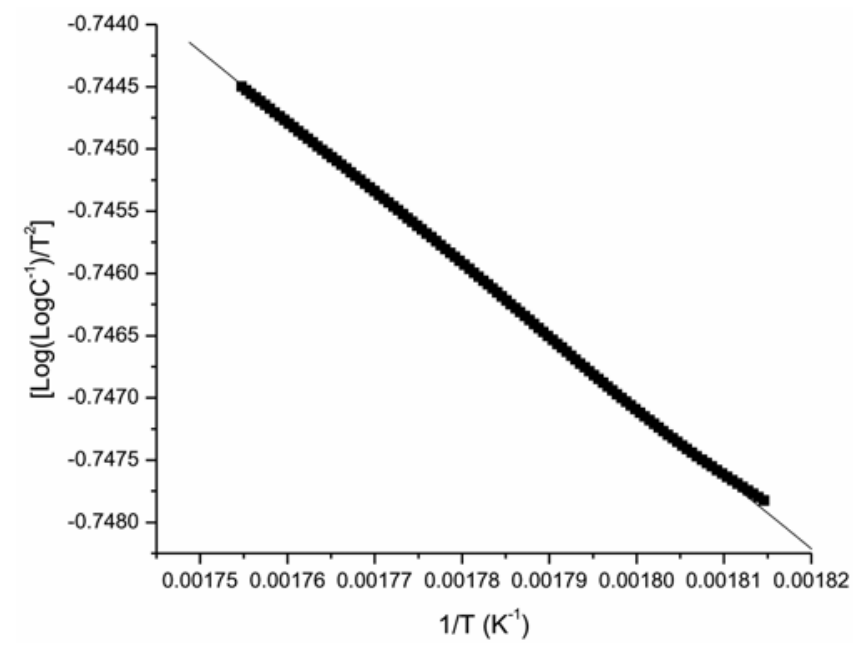

Figure 7. Plot of best fitting for stage 2 (547-797 K).

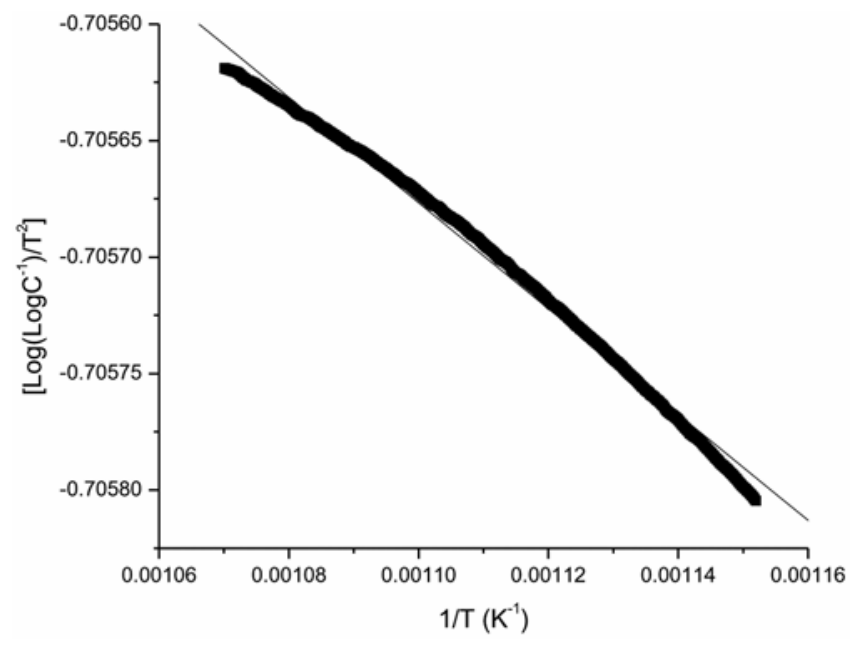

Figure 8. Plot of best fitting for stage 3 (1039-1113 K). respectively reducing the final product to strontium acetylene dicarboxylate. The losses corresponding to these decomposition stages are further supported by the DTA peaks shown in figure 5. The non-isothermal kinetic parameters have been worked out for the decomposition stages between $379-445 \mathrm{~K}, 547-797 \mathrm{~K}$ and $1039-1113 \mathrm{~K}$ using the integral method by applying Coats-Redfern (1964) approximation method.

$$
\log \left(\log C^{-1} / T^{2}\right)=\log Z R / \beta E-E / 2 \cdot 303 R T,
$$

where $C=\left(w_{\mathrm{oc}}-w\right) / w_{\mathrm{oc}}, w_{\mathrm{oc}}$ is the total mass loss for a particular stage, $w$ the mass loss at absolute temperature $T, Z$ the pre-exponential factor and $\beta$ the heating rate. The slope between $\log \left(\log C^{-1} / T^{2}\right)$ and $1 / T$ gives the value of activation energy. The theoretical and experimental values fitted for the three stages of decomposition in the temperature ranges of $379-445 \mathrm{~K}, 547-797 \mathrm{~K}$ and 1039 $1113 \mathrm{~K}$ are shown in figures 6-8. The slopes of these curves yield the value of activation energies. The calculated activation energies from the slope of the curves for the three decomposition stages are $277.10 \mathrm{~kJ} / \mathrm{mol}$, $290.38 \mathrm{~kJ} / \mathrm{mol}$ and $125 \mathrm{~kJ} / \mathrm{mol}$ with corresponding frequencies $2.3 \times 10^{5}, 1.03 \times 10^{7}, 2.65 \times 10^{3}\left(\mathrm{~s}^{-1}\right)$ in the temperature ranges, $379-445 \mathrm{~K}, 547-797 \mathrm{~K}$ and 1039 $1113 \mathrm{~K}$, respectively.

\section{Discussion}

From the dielectric studies as described above it is clear that the transition occurs in the region $360<T<390 \mathrm{~K}$ $\left(87-117^{\circ} \mathrm{C}\right)$. To account for this transition, two possibilities could be considered. One is that it may be thermally unstable due to ejection of water molecules at different stages of temperature. The thermal stability of the material is revealed in figure 5. It is clear that initially the material loses three water molecules in the temperature range of about $106-172^{\circ} \mathrm{C}$ and then in the second stage, it loses three water molecules in the temperature range of about $274-524^{\circ} \mathrm{C}$ and thereafter, the changes that occur suggest a decomposition process, whereby the parent material loses its originality and no longer remains strontium tartrate. However, in the temperature range of about $106-274^{\circ} \mathrm{C}$ the material maintains itself to be strontium tartrate. Therefore, the characteristic features of strontium tartrate will be retained only up to a maximum of about $274^{\circ} \mathrm{C}$. It shows a dielectric characteristic suggesting a phase transition. There can be two possibilities to account for this behaviour. One could be losing of three water molecules in the temperature range of $106-172^{\circ} \mathrm{C}(379-$ $445 \mathrm{~K}$ ). The other could be suggestive of the material being ferroelectric similar to calcium or cadmium tartrate as reported in the literature (Gon 1990; Toress et al 1998). Toress et al reported two phase transitions in cadmium tartrate crystals, one corresponding to structural changes and the other due to loss of water. Here, in the 
present case of strontium tartrate, the only transition that is observed is at about $97^{\circ} \mathrm{C}$. A close examination of the DTA curve of figure 5 also suggests the initiation and end of the endotherms in the temperature range $380-480 \mathrm{~K}$ (corresponding to $107-207^{\circ} \mathrm{C}$ ) which supports the conjecture that a crystallographic change due to polymorphic phase transition may be occurring in the material (including the change as a result of loss of water molecules) which leads to the dielectric anamoly at around $97^{\circ} \mathrm{C}$ or so. Similar such observation has been reported for gadolinium tartrate crystals (Want et al 2006). Based on what is reported in the literature and the results obtained here as described above, it may be suggested that the material under study exhibits a transition behaviour in the temperature range of $87<T<117^{\circ} \mathrm{C}$.

The dielectric constant, $\varepsilon^{\prime}$, is related to polarization of materials and four primary mechanisms of polarization include electronic polarization, $\alpha_{\mathrm{e}}$, atomic or ionic polarization, $\alpha_{\mathrm{i}}$, dipolar or orientational polarization, $\alpha_{\mathrm{o}}$ and interfacial polarization. The $\alpha_{\mathrm{e}}$ occurs at higher frequencies of the order of $10^{15} \mathrm{~Hz}$ which falls in the ultra violet optical frequency range. At frequencies in the infrared range $\left(10^{12}-10^{13} \mathrm{~Hz}\right)$, atomic and ionic polarization takes place. Dipolar polarization or orientational polarization contributes to the dielectric properties in sub infrared range of frequencies $\left(10^{3}-10^{6} \mathrm{~Hz}\right)$ at room temperature. The interfacial polarization occurs when mobile charge carriers are impeded by a physical barrier that inhibits charge migration. The charges get piled up at the barrier resulting into a localized polarization in the material. The frequency range of sensitivity for interfacial polarization is in the low frequency range and may extend into the kilocycles $\left(10^{3} \mathrm{~Hz}\right)$ range (Hench and West 1990). Space charge (or diffusing) polarization involves a limited transport of charge carriers until they are stopped at a potential barrier which could be a grain boundary or phase boundary. Space charge and dipolar polarization are relaxation processes and are strongly temperature dependent whereas electronic or ionic polarizations are resonance processes and almost temperature independent (Moulson and Herbert 1990). The frequency dependence of $\varepsilon^{\prime}$ also suggests which of the polarization mechanisms are effective in a given situation. At very low frequencies the dielectric characteristics are influenced by interfacial polarization or at the microscopic level by ionic or space charge polarization. As the frequency is increased the space charge, orientational and ionic contributions get eliminated and finally the contribution due to electrons remain.

Dependence of dielectric loss on frequency also can lead to polarization mechanisms which are called into play. The dielectric loss peak also appears at a frequency where one or the other polarization mechanisms start damping out and as such the individual contribution can be determined by analyzing the dielectric properties either as a function of frequency or temperature.
The sharp rise of $\varepsilon^{\prime}$ at around $370 \mathrm{~K}$, attainment of its maximum value of 25.5 at $370 \mathrm{~K}$ corresponding to a frequency of $100 \mathrm{~Hz}$ followed by its sudden fall, suggests $370 \mathrm{~K}$ to be the transition temperature, $T_{\mathrm{c}}$. The maximum value of $\varepsilon^{\prime}$ at $370 \mathrm{~K}$ falls down to much lower values as the frequency increases $(f>100 \mathrm{~Hz})$, but the $T_{\mathrm{c}}$ remains the same at all the frequencies. The $\varepsilon^{\prime}$ is independent of temperature till near about $360 \mathrm{~K}$ irrespective of the frequency of the applied field $(100<f<700 \mathrm{kHz})$ which indicates that no new polarization mechanism is called into play. The contributions from new polarization processes are called into play only when the temperature exceeds $360 \mathrm{~K}$. Ionic and electronic polarization being essentially resonance processes are, therefore, temperature independent. However, the other two polarizations viz. orientational (dipolar) and space charge being relaxation processes are strongly dependent on temperature (Moulson and Herbert 1990).

The contribution towards polarization from space charge effects depends on purity and perfection of crystals because the impurities or other forms of imperfection create potential barriers, limiting the transport of charge carriers and hence increasing the value of $\varepsilon^{\prime}$. The contribution from space charge towards polarization mechanism at lower temperature and higher frequency is insignificant as to make it negligible. However, as the temperature increases, the contribution from the space charge towards polarization may have a tendency to increase. The dipolar polarization being inversely proportional to temperature (Omar 1975), it decreases as the temperature rises.

In the present case, $\varepsilon^{\prime}$ and the dielectric loss, are observed to be dependent on temperature and frequency of the applied a.c. field; the value of $\varepsilon^{\prime}$ increases with the decrease in frequency at all the temperatures in the range $100-600 \mathrm{~K}$. The value of $\varepsilon^{\prime}$ attained at a particular temperature for a particular frequency remains the same (independent of temperature) except in the vicinity of the transition temperature where its value touches the highest value and then returns to its original value. In other words, $\varepsilon^{\prime}$ maintains a constant value corresponding to a particular frequency in the temperature range $T<T_{\mathrm{c}}$ and $T>T_{\mathrm{c}}$. It means that the $\varepsilon^{\prime}$ is frequency dependent but temperature independent in pre or post $T_{\mathrm{c}}$ range. It means that in this particular case the contribution towards polarization comes from ionic or space charge at lower frequencies which get eliminated at higher frequencies.

\section{Conclusions}

From the above observations and discussion one may conclude the following:

(I) The dielectric parameters viz. $\varepsilon^{\prime}$ and $\tan \delta$, depend on both temperature as well as frequency of the applied a.c. field. The value of $\varepsilon^{\prime}$ is independent of temperature till 
around $360 \mathrm{~K}$ at all the frequencies $(100-700 \mathrm{kHz})$ of the applied a.c. field. It however, increases abruptly and achieves a peak value of 25.5 at $100 \mathrm{kHz}$. The peak value of $\varepsilon^{\prime}$ is strongly dependent on frequency (ranging between 100 and $700 \mathrm{kHz}$ ). On attaining the peak value, in the temperature range $87<T<117^{\circ} \mathrm{C}$, the value of $\varepsilon^{\prime}$ falls suggesting a transition at around $102^{\circ} \mathrm{C}$.

(II) $\varepsilon^{\prime}$ maintains a constant value corresponding to any particular frequency ranging between 100 and $700 \mathrm{kHz}$ in the temperature $T<T_{\mathrm{c}}$ and $T>T_{\mathrm{c}}$ meaning thereby that the $\varepsilon^{\prime}$ is frequency dependent but temperature independent in the pre and post $T_{\mathrm{c}}$ range $87<T<117^{\circ} \mathrm{C}$. It suggests that the contribution towards polarization may be due to ionic or space charge polarization which gets eliminated at higher frequencies.

(III) The thermograms suggest that strontium tartrate pentahydrate is thermally stable up to $105^{\circ} \mathrm{C}$ beyond which it loses three water molecules, two intrinsic water molecules and then one intrinsic water molecule at three different temperature intervals till finally at around $840^{\circ} \mathrm{C}$ it gets reduced to strontium acetylene tricarboxylate.

(IV) Attainment of peak value of $\varepsilon^{\prime}$ at around $100^{\circ} \mathrm{C}$ or so followed by an abrupt fall suggests it to be a ferroelectric transition which is supported by the results of thermoanalytical studies. Thermal and dielectric studies suggest that crystallographic change due to polymorphic phase transition may be occurring in the material (including the change due to loss of water molecules) which leads to the dielectric anamoly at around $100^{\circ} \mathrm{C}$ or so.

(V) Application of Coats-Redfern approximation method for obtaining non-isothermal kinetic parameters yield the values of activation energies corresponding to three decomposition stages of STP as $277 \cdot 10 \mathrm{~kJ} / \mathrm{mol}, 290 \cdot 38 \mathrm{~kJ} / \mathrm{mol}$ and $125 \mathrm{~kJ} / \mathrm{mol}$ with corresponding frequencies $2 \cdot 3 \times 10^{5}$, $1.03 \times 10^{7}, 2.65 \times 10^{3}\left(\mathrm{~s}^{-1}\right)$ in the temperature ranges $379-445 \mathrm{~K}, 547-797 \mathrm{~K}$ and $1039-1113 \mathrm{~K}$, respectively.

\section{Acknowledgements}

One of the authors (AF) is thankful to Dr A R Wanchoo, Director, NIT, Srinagar, for his support. The corresponding author (PNK) is thankful to the AICTE, New Delhi, for the award of an Emeritus fellowship. The authors are also thankful to Prof. Naresh Padha, Department of Physics and Electronics, University of Jammu, Jammu, for his kind support during the research work.

\section{References}

Arora S K, Patel V, Patel R G, Amin B and Kothari A $2004 \mathrm{~J}$. Phys. Chem. Solids 65965

Arora S K, Patel V, Chudasama B and Amin B 2005 J. Cryst. Growth 275657

Arora S K, Patel V, Kothari A and Amin B 2004 Cryst. Growth Des. 4343

Arora S K, Patel V, Kothari A and Amin B 2004 Bull. Mater. Sci. 27141

Arora S K, Patel V, Kothari A and Amin B 2005 J. Am. Ceram. Soc. 883469

Coats A W and Redfern J P 1964 Nature 20168

Fousek F, Cross L E and Seely K 1970 Ferroelectrics 163

Gon H B 1990 J. Cryst. Growth 102501

Hench L L and West J K 1990 Principles of electronic ceramics (New York: John Wiley and Sons)

Henisch HK 1973 Crystal growth in gels (University Park, Ohio: Pennsylvania University Press)

Henisch H K 1988 Crystal growth in gels and liesgang rings (Cambridge: Cambridge University Press)

Ivanov N R 1984 Ferroelectr. Lett. 2745

Jona F and Shirane G 1993 Ferroelectric crystals (New York: Dover Publications, Inc), Ch. VII

Moulson A J and Herbert JM 1990 in Electroceramicsmaterials, properties, applications (New York: Chapman and Hall)

Omar M A 1975 in Elementary solid state physics - principles and applications (Massachusetts, USA: Eddison-Wesley Publishing Company)

Rahimkutty M H, Rajendra Babu K, Shreedharan Pillai K, Sudarshankumar M R and Nair C M K 2001 Bull. Mater. Sci. 24249

Suryanarayana K and Dharmaprakash S M 2000 Mater. Lett. 42 92

Suryanarayana K and Dharmaprakash S M 2002 Mater. Chem. Phys. 77179

Toress ME, Lopez T, Peraza J, Stockel J, Yanes A C, Gonzalez-Silgo C, Ruiz-Perez C and Lorenzo-Luis P A 1998 J. Appl. Phys. 845729

Toress M E, Stockel J, Peraza J F and Yanes A C 1995 J. Cryst. Growth 156421

Toress ME, Lopez T, Peraza J, Stockel J, Yanes A C, Gonzalez-Silgo C, Solan X, Garcia-Valle's M, Want B, Ahmad F and Kotru P N 2006 Mater. Sci. \& Engg. A431 237 